\title{
Immunisation status and determinants of left-behind children aged 12-72 months in central China
}

\author{
Z.L. NI, X.D TAN*, H.Y. SHAO AND Y. WANG \\ School of Public Health, Wuhan University, Wuhan 430071, Hubei, China
}

Received 26 April 2016; Final revision 27 December 2016; Accepted 22 February 2017; first published online 30 March 2017

\section{SUMMARY}

Many parents move from rural China to urban areas in search of job opportunities, and leave their children behind to be raised by relatives. We aimed to assess the immunisation coverage, including the 1:3:3:3:1 vaccine series (one dose of Bacilli Chalmette-Guérin vaccine; three doses of live attenuated oral poliomyelitis vaccine; three doses of diphtheria, tetanus and pertussis combined; three doses of hepatitis B vaccine; and one dose of measles-containing vaccine), in children aged 12-72 months and identify the determinants of immunisation uptake among left-behind children in Hubei Province, Central China, in 2014. In this cross-sectional study using the World Health Organization's cluster sampling technique, we surveyed 1368 children from 44 villages in 11 districts of Hubei Province. The socio-demographic and vaccination status data were collected by interviewing primary caregivers using a semi-structured questionnaire and reviewing the immunisation cards of the children. Univariate and multivariate analyses were used to identify the determinants of complete vaccination and age-appropriate vaccination. For each dose of the five vaccines, the vaccination coverage in the left-behind and non-left-behind children was $>90 \%$; however, the age-appropriate vaccination coverage for each vaccine was lower in left-behind than in non-left-behind children. For the five vaccines, the fully vaccinated rate of left-behind children were lower than those of non-left-behind children $(89 \cdot 1 \%, 92 \cdot 7 \% ; P=0.013)$ and age-appropriate immunisation rate of left-behind children were lower than those of nonleft-behind children $(65 \cdot 7 \%, 79 \cdot 9 \% ; P<0 \cdot 001)$. After controlling for potential confounders, we found that the parenting pattern, annual household income and attitude of the primary caregiver towards vaccination significantly influenced the vaccination status of children. Moreover, we noted a relatively high prevalence of delayed vaccination among left-behind children. Hence, we believe that the age-appropriate immunisation coverage rate among left-behind children in rural areas should be further improved by delivering and sustaining primary care services.

Key words: Determinants, immunisation (vaccination), left-behind children.

\section{INTRODUCTION}

The migration of labour is a crucial consequence of economic development in many countries. An increasing number of adults migrate to urban areas to seek job opportunities in these locations [1]. Over the last decade, China has achieved marked economic growth through development of the market economy [2]. As in other countries, there is a large regional income

\footnotetext{
* Author for correspondence: X. Tan, School of Public Health, Wuhan University, Wuhan 430071, Hubei, China. (Emails: xiaodongtan0604@sina.com, xiaodongtan@yahoo.com)
} 
imbalance has developed in China, which remains the key factor driving massive internal migration. The number of rural-to-urban migrants increased from 77 million in 1990 to 221 million in 2010, thus accounting for, $16 \cdot 5 \%$ of the total population of China [3]. As most of these migrant workers perform low-paying jobs, they usually leave behind close relatives, including children, spouses and parents. Thus, a large number of children in rural areas are increasingly becoming 'left-behind', and are raised by relatives [4].

Parental migration from rural to urban areas often leads to higher incomes and enhanced socio-economic status. However, reduced parental care, in the absence of one or both parents, negatively influences development in children [5]. The adverse effects of parental separation over an extended period on the psychological, behavioural and educational outcomes have been characterised in several studies. However, only a few studies have examined the immunisation status and determinants of left-behind children in rural areas.

Communicable diseases have major impacts on health among young children, particularly in lessdeveloped areas. At present, hepatitis B and tuberculosis are significant causes of mortality and morbidity in children [6]. Vaccination is the most effective measure for protecting children from infectious diseases [7]. The immunisation of a child lowers the likelihood of infecting other children, family members or closely connected individuals [8]. In fact, immunisation is the most cost-effective health intervention worldwide. The World Health Organization (WHO) estimated that $17 \%$ of global annual under-five mortality could be prevented by increasing routine vaccination coverage, including $5.4 \%$ through pneumococcal vaccination, $5 \cdot 2 \%$ through rotavirus vaccination, $2 \cdot 3 \%$ through Hib pertussis vaccination, $2 \cdot 2 \%$ through pertussis vaccination, $1.3 \%$ through measles vaccination and $0.7 \%$ through tetanus vaccination [7]. However, immunisation in children is impeded by many factors, such as education and low parental knowledge [9], late birth order [10], family income [11] and even population mobility [12].

The National Immunization Program (NIP) was established in China in 1978. The 1:3:3:3:1 series includes a birth dose of Bacilli Calmette-Guérin (BCG) vaccine, three doses of oral poliovirus vaccine (OPV1 at 2 months, OPV2 at 3 months and OPV 3 at 4 months), three doses of diphtheria-tetanus-pertussis vaccine (DTP1 at 3 months, DTP2 at 4 months and DTP3 at 5 months); three doses of hepatitis B vaccine (HepB1 at birth, HepB2 at 1 month and HepB3 at
6 months), and one dose of measles-containing vaccine (MV at 8 months), it is purchased by the government and provided to all children free of charge, without any extra service fees. According to the National Immunization Coverage Survey conducted in 2012, the coverage of these five types of vaccines of children aged 12-24 months are 99.79\%, 99.74\%, $99 \cdot 44 \%, 99 \cdot 42 \%$ and $99 \cdot 49 \%$, respectively, in China; the coverage of complete vaccination (all five vaccines) was $98.74 \%$ [13]. However, the problem of low immunisation coverage of left-behind children should be clearly recognised.

Hubei is a big multi-ethnic province with a large fluid population. It is located in the middle part of China, and plays an important part in economic and social development. Hubei Province is also a major agricultural province in the middle part of China, with $70 \%$ of the population residing in rural areas. Many rural labourers from Hubei Province acquire jobs in other areas. Consequently, approximately $30 \%$ of children in Hubei Province are left behind by parents to be raised by grandparents or other relatives. Therefore, a study of the vaccination coverage of the five basic vaccines among left-behind children in Hubei Province is important.

In the present study, we aimed to assess the age-appropriate coverage of the five vaccines among the left-behind children and non-left-behind children in Hubei, and explore the determinants associated with complete and age-appropriate immunisation coverage in left-behind children.

\section{METHODS}

\section{Target population}

In this study, the target population included children, who were born between 1 January 2008 and 31 December 2013 in Hubei Province, and their primary caregivers. Children were categorized into left-behind and non-left-behind children. We defined a left-behind child as a child residing in the family household for a period of at least 6 months with parents who were migrant workers currently working away from their hometown for a period of at least 6 months. Children who had been residing in the province for $<3$ months were excluded. Based on the guidelines of China's Ministry of Health on the evaluation of immunisation rate, primary immunisation was considered as age-appropriate vaccination with the five types of vaccines at the required doses within the first 12 
months. In rural areas, most children aged 72 months or more would enter primary schools and should be excluded to avoid the impact on our results of immunisation requirements of the primary schools.

In the present study, a primary caregiver was defined as a person responsible for caring for the leftbehind child on a daily basis, for a period of at least 6 months, which comprised activities such as arranging daily schedules, preparing or ensuring access to meals, taking the child for immunisation, looking after the child when he/she is sick.

\section{Sample design and investigations}

A cross-sectional study was conducted using the multi-stage stratified sampling method based on the WHO-advocated cluster sampling technique [14]. In China, the smallest administrative rural population unit is the 'village unit'. In the first stage, a total of 103 counties in Hubei Province were stratified by terrain: plains, hills and mountains. Thereafter, nine districts, including three districts from each terrain, were identified from the 103 districts via simple random selection. In the second stage, four villages were randomly selected from each district. Accordingly, 36 villages were selected as clusters. By reviewing the measles vaccination coverage of the children aged 0 6 years in Hubei Province in 2010 (86\%) [15], using a desired precision of $\pm 5 \%$ with $95 \%$ confidence intervals, and assuming a design effect of 2 , we obtained the required number of surveyed children per cluster for a variable number of clusters, according to the table recommended by the WHO manual [14]. The final sample size was determined as a total of 31 children per cluster for 36 clusters. Thus, the final population included 1116 children in nine districts covering 72 villages. The sampling weights were calculated for each county based on the distribution of leftbehind and non-left-behind children. One household per sampled village was chosen as the starting point, and the investigators were divided into four groups for conducting household surveys from the centre of the village to all directions in concentric circles.

Information on the demographic and socioeconomic characteristics (age, gender, education, occupation, income, ethnicity, attitudes towards vaccination, distance from home to the immunisation centre, etc.) of the primary caregivers was collected via a face-to-face interviewer-administered questionnaire. Immunisation status data were obtained by reviewing the child's home immunisation certificate or by reviewing the child's immunisation card at the immunisation station if the certificate was not available. If neither were available, the registry of the village doctor was reviewed, and the names of the children and parents, as well as the birth date of the children, were recorded after surveying the essential information. Moreover, the immunisation status was assessed online via the system of the expanded NIP in Hubei Province to complete the questionnaires. In the absence of any information, the child's immunisation status was recorded as 'unvaccinated' or 'unknown'. Moreover, to explore the reason why the children were not fully vaccinated, we also examined the caregivers whose children were not fully vaccinated.

\section{Variable definitions and data analysis}

There were two outcomes in the present study: (1) the full vaccination rate was considered in children who received all the vaccine series at the required doses, regardless of the age at each dose, whereas the incomplete vaccination rate was considered in children who did not receive any vaccinations or did not receive all the doses for each vaccination; and (2) the age-appropriate immunisation rate was defined as the percentage of children who received all the vaccines at the required dose and at the appropriate age.

The individual-level variables included parenting style (both-parenting, grand-parenting or singleparenting); gender (male, female); and HuKou (rural, urban); only child (yes, no). The householdlevel variables included parenting style, caregiver education level, caregiver occupation, family income (the annual income of all family members), caregiver attitude and knowledge of vaccination. The sociodemographic variables comprised terrain (plains, mountains and hills); economic condition (developed, middle and underdeveloped); and residential status (local and recurrent population).

All data were individually uploaded twice to the database using Expanded Program on Immunization (EPI) Data 3.02 software, and were reviewed by a different person. Statistical analyses were performed using SPSS (Statistical Package for Social Sciences) version 21.0 and Excel 2007 software. By using complete vaccination as a reference, statistical analysis, including descriptive analysis, was performed to determine the coverage of various vaccines in left-behind and non-left-behind children. The $\chi^{2}$ tests were used to evaluate the relationship between vaccination status 
and each factor. A stepwise regression method for two-way selection was used to repeatedly exclude nonsignificant variables, thus yielding a local optimum regression equation. Multivariate forward logistic regressions were used to identify the factors independently associated with complete vaccination and the timeliness of vaccination.

\section{RESULT}

\section{Socio-demographic characteristics}

A total of 1368 children and their primary caregivers participated in the study. The children had different primary caregivers, including both parents in 725 (52.99\%) non-left-behind children, grandparents in $366(26.72 \%)$ left-behind children with both parents working outside the village and 278 (20.29\%) left-behind children with one parent working outside the village.

A similar gender disaggregation profile was observed in both non-left-behind and left-behind children $(55 \cdot 85 \%$ male and $44 \cdot 15 \%$ female). At the time of the survey, $36 \cdot 62 \%$ children were aged $12-24$ months, whereas $34 \cdot 72 \%$ children were aged $25-48$ months. Moreover, 623 children $(45 \cdot 54 \%)$ were the only child in the household. More than half of the caregivers had attended middle school $(58 \cdot 26 \%)$, and a relatively small proportion $(7 \cdot 38 \%)$ received a high school education or greater. The other sociodemographic characteristics of the participants are listed in Table 1.

\section{Immunisation coverage for each dose of the primary course in left-behind and non-left-behind children}

The immunisation rate for each dose of the five vaccines is listed in Table 2. The first-dose hepatitis B vaccination rates among left-behind and non-left-behind children were the highest $(98.2 \%$ and $98.7 \%$, respectively). The immunisation rates for HepB, BCG, OPV, DPT, and MCV ranged from $90 \cdot 8 \%$ to $97 \cdot 5 \%$ in left-behind children and $92 \cdot 1 \%$ to $98.7 \%$ in non-left-behind children. Non-left-behind children $(92.7 \%)$ were more likely to receive complete vaccination as compared with left-behind children (79.9\%); the difference was statistically significant $\left(\chi^{2}=35 \cdot 2\right.$, $P<0.001)$. Moreover, the coverage for each of the five vaccines decreased with each subsequent dose. The coverage gap between the first and third dose of HepB ranged from $6.7 \%$ among left-behind children to $4.6 \%$ among non-left-behind children. Moreover, for the all five vaccines, the age-appropriate immunisation rates of all children were lower than the vaccination coverage. In addition, the age-appropriate immunisation rates for almost all the doses exhibited significant differences between left-behind and non-left-behind children.

\section{Determinants of complete vaccination and age-appropriate vaccination}

Multivariate forward logistic regression was used to identify the factors independently associated with the complete immunisation and age-appropriate immunisation. Multinomial logistic regression analyses indicated significant differences in the coverage of full immunisation status and the timeliness of vaccination, parenting style, age, caregiver education, annual household income, awareness of the importance of immunisation, knowledge of the vaccination programme and satisfaction with immunisation station service. The results of multivariate forward logistic regression showed that, compared with the non-left-behind children (both-parenting), children with single-parenting and grand-parenting styles were less likely to receive complete vaccination and more likely to receive delayed immunisation. Compared with children aged 1-2 years, children aged 3-6 years exhibited lower complete vaccination and age-appropriate immunisation rate. An education level of middle school or high school and above increased the likelihood of receiving complete immunisation and age-appropriate immunisation. Moreover, an increase in household income had a positive influence on vaccination coverage and age-appropriate immunisation. If the primary caregiver was aware of the importance and schedule of immunisation, the child was more likely to be fully vaccinated and receive age-appropriate immunisation.

The results of multivariate forward logistic regression with OR values of children receiving full immunisation and age-appropriate immunisation are listed in Table 3 .

\section{DISCUSSION}

The EPI has been implemented for 9 years in China, since 2005, and has eliminated the fee for immunisation services; thus, the immunisation coverage has improved. However, the incidence of some related infectious diseases remains high, and the outbreak of epidemics, such as that of measles, remains an actual 
Table 1. Socio-demographic characteristics of participants in our study

\begin{tabular}{|c|c|c|c|c|c|c|}
\hline & \multicolumn{2}{|c|}{ Left behind } & \multicolumn{2}{|c|}{ Not left behind } & \multicolumn{2}{|l|}{ Total } \\
\hline & $N$ & $\%$ & $N$ & $\%$ & $N$ & $\%$ \\
\hline \multicolumn{7}{|l|}{ Parenting style } \\
\hline Single-parenting & 278 & $43 \cdot 23$ & - & - & - & - \\
\hline Grand-parenting & 365 & $56 \cdot 77$ & - & - & - & - \\
\hline \multicolumn{7}{|l|}{ Gender } \\
\hline Boy & 352 & $54 \cdot 74$ & 412 & $56 \cdot 83$ & 764 & $55 \cdot 85$ \\
\hline Girl & 291 & $45 \cdot 26$ & 313 & $43 \cdot 23$ & 604 & $44 \cdot 15$ \\
\hline \multicolumn{7}{|l|}{ Child's age (months) } \\
\hline $12-24$ & 235 & $36 \cdot 55$ & 307 & $42 \cdot 34$ & 542 & $39 \cdot 62$ \\
\hline $25-48$ & 211 & $32 \cdot 81$ & 264 & $36 \cdot 41$ & 475 & $34 \cdot 72$ \\
\hline $49-72$ & 197 & $30 \cdot 64$ & 154 & $21 \cdot 24$ & 351 & $25 \cdot 66$ \\
\hline \multicolumn{7}{|l|}{ Only child } \\
\hline Yes & 280 & $43 \cdot 55$ & 343 & $47 \cdot 31$ & 623 & $45 \cdot 54$ \\
\hline No & 363 & $56 \cdot 45$ & 382 & $52 \cdot 69$ & 745 & $54 \cdot 46$ \\
\hline \multicolumn{7}{|l|}{ Caregiver's education } \\
\hline No schooling & 97 & 15.09 & 98 & $13 \cdot 52$ & 195 & $14 \cdot 25$ \\
\hline Elementary school & 126 & $19 \cdot 60$ & 149 & $20 \cdot 55$ & 275 & $20 \cdot 10$ \\
\hline Middle school & 378 & 58.79 & 419 & $57 \cdot 79$ & 797 & $58 \cdot 26$ \\
\hline High school and above & 42 & 6.53 & 59 & $8 \cdot 14$ & 101 & $7 \cdot 38$ \\
\hline \multicolumn{7}{|l|}{ Caregiver's age (years) } \\
\hline$<30$ & 110 & $17 \cdot 11$ & 322 & $44 \cdot 41$ & 432 & $31 \cdot 58$ \\
\hline $31-40$ & 121 & $18 \cdot 82$ & 281 & 38.76 & 402 & $29 \cdot 39$ \\
\hline $41-50$ & 124 & $19 \cdot 28$ & 76 & $10 \cdot 48$ & 200 & $14 \cdot 62$ \\
\hline$>51$ & 288 & $44 \cdot 79$ & 46 & $6 \cdot 34$ & 334 & $24 \cdot 42$ \\
\hline \multicolumn{7}{|c|}{ Annual household income (Yuan per year) } \\
\hline$<5000$ & 121 & $18 \cdot 82$ & 160 & $22 \cdot 07$ & 281 & $20 \cdot 54$ \\
\hline $5000-8000$ & 323 & $50 \cdot 23$ & 359 & $49 \cdot 52$ & 682 & $49 \cdot 85$ \\
\hline$>8000$ & 199 & $30 \cdot 95$ & 206 & $28 \cdot 41$ & 405 & $29 \cdot 61$ \\
\hline \multicolumn{7}{|l|}{ Terrain } \\
\hline Plain area & 283 & $44 \cdot 01$ & 332 & $45 \cdot 79$ & 615 & 44.96 \\
\hline Hilly area & 151 & $23 \cdot 48$ & 165 & $22 \cdot 76$ & 316 & $23 \cdot 10$ \\
\hline Mountainous area & 209 & $32 \cdot 50$ & 228 & $31 \cdot 45$ & 473 & 31.94 \\
\hline \multicolumn{7}{|c|}{ Awareness of the importance of immunisation } \\
\hline No & 122 & 18.97 & 133 & $18 \cdot 34$ & 255 & $18 \cdot 64$ \\
\hline Yes & 521 & $81 \cdot 03$ & 592 & $81 \cdot 66$ & 1113 & $81 \cdot 36$ \\
\hline \multicolumn{7}{|c|}{ Knowledge of the vaccination programme } \\
\hline No & 269 & $41 \cdot 84$ & 288 & $39 \cdot 72$ & 557 & $40 \cdot 72$ \\
\hline Yes & 374 & $58 \cdot 16$ & 437 & $60 \cdot 28$ & 811 & $59 \cdot 28$ \\
\hline \multicolumn{7}{|c|}{ Satisfied with the immunisation station service } \\
\hline No & 76 & $11 \cdot 82$ & 90 & $12 \cdot 41$ & 166 & $12 \cdot 13$ \\
\hline Yes & 567 & $88 \cdot 18$ & 635 & $87 \cdot 59$ & 1202 & $87 \cdot 87$ \\
\hline Total & 643 & $47 \cdot 01$ & 725 & $52 \cdot 99$ & 1368 & 100 \\
\hline
\end{tabular}

threat [6]. From 2006 to 2012, Hubei experienced nine outbreaks of measles with 209 cases [16]. From 2005 to 2014, in Hubei, there are 219 outbreaks of mumps with 9213 cases [17]. Hence, it is vital to understand the actual vaccination status of the children and assess the behaviour and beliefs of parents towards vaccination.

Due to rapid economic growth and urbanisation, China has recently experienced unprecedented urbanisation and changes in the structure of society [4]. The number of individuals migrating from rural areas to urban areas is markedly increasing. The low vaccination coverage of migrant children has been examined in China and other developing countries [18]. However, the low immunisation rate and low age-appropriate immunisation rate of left-behind children remains unclear. The differences in the immunisation rate, particularly the age-appropriate 
Table 2. Recommended childhood primary immunisation schedule in China and coverage of the immunisation status for each vaccine (\%)

\begin{tabular}{|c|c|c|c|c|c|c|c|c|}
\hline \multirow[b]{2}{*}{ Vaccine 1} & \multicolumn{2}{|c|}{ Vaccination coverage $\%$} & \multirow[b]{2}{*}{$\chi^{2}$} & \multirow[b]{2}{*}{$P$} & \multicolumn{2}{|c|}{$\begin{array}{l}\text { Age-appropriate vaccination } \\
\text { coverage } \%\end{array}$} & \multirow[b]{2}{*}{$\chi^{2}$} & \multirow[b]{2}{*}{$P$} \\
\hline & L-b children & N-1-b children & & & L-b children & N-1-b children & & \\
\hline BCG & $97 \cdot 5$ & $98 \cdot 7$ & $3 \cdot 832$ & $0 \cdot 041$ & $92 \cdot 1$ & $93 \cdot 5$ & $1 \cdot 076$ & $0 \cdot 176$ \\
\hline OPV1 & $95 \cdot 5$ & $96 \cdot 8$ & $1 \cdot 322$ & $0 \cdot 125$ & $82 \cdot 6$ & $86 \cdot 7$ & $4 \cdot 611$ & 0.019 \\
\hline OPV2 & $92 \cdot 4$ & $94 \cdot 5$ & $2 \cdot 474$ & 0.072 & $81 \cdot 7$ & $85 \cdot 8$ & $4 \cdot 322$ & 0.023 \\
\hline OPV3 & $91 \cdot 3$ & $92 \cdot 1$ & $0 \cdot 221$ & $0 \cdot 319$ & $77 \cdot 4$ & $82 \cdot 5$ & $5 \cdot 420$ & 0.021 \\
\hline DPT1 & $93 \cdot 7$ & $94 \cdot 1$ & $03 \cdot 22$ & $0 \cdot 319$ & $73 \cdot 5$ & $81 \cdot 2$ & $11 \cdot 575$ & $<0 \cdot 001$ \\
\hline DPT2 & $91 \cdot 7$ & $93 \cdot 2$ & $0 \cdot 117$ & $0 \cdot 409$ & $79 \cdot 9$ & $86 \cdot 7$ & $11 \cdot 537$ & $<0.001$ \\
\hline DPT3 & $90 \cdot 8$ & $93 \cdot 1$ & $1 \cdot 085$ & $0 \cdot 174$ & $75 \cdot 4$ & $81 \cdot 2$ & $6 \cdot 826$ & $0 \cdot 005$ \\
\hline НерB1 & $98 \cdot 2$ & 98.9 & $1 \cdot 047$ & $0 \cdot 193$ & $91 \cdot 1$ & $96 \cdot 5$ & $17 \cdot 742$ & 0.023 \\
\hline НерB2 & $93 \cdot 2$ & $94 \cdot 5$ & $1 \cdot 039$ & $0 \cdot 182$ & $81 \cdot 5$ & $91 \cdot 7$ & $23 \cdot 386$ & $<0 \cdot 001$ \\
\hline HepB3 & $91 \cdot 5$ & $94 \cdot 3$ & 3.955 & 0.023 & $80 \cdot 1$ & $90 \cdot 3$ & $29 \cdot 391$ & $<0 \cdot 001$ \\
\hline MV & $91 \cdot 5$ & 93.9 & $2 \cdot 403$ & 0.037 & $70 \cdot 5$ & $80 \cdot 2$ & $17 \cdot 328$ & $<0 \cdot 001$ \\
\hline All-course & $89 \cdot 1$ & $92 \cdot 7$ & $5 \cdot 320$ & $0 \cdot 013$ & $65 \cdot 7$ & $79 \cdot 9$ & $35 \cdot 162$ & $<0 \cdot 001$ \\
\hline
\end{tabular}

BCG, Bacilli Calmette-Guérin vaccine; OPV, oral poliovirus vaccine; DPT, diphtheria-tetanus-pertussis vaccine; HepB, hepatitis B vaccine; MV, measles-containing vaccine; L-b, left-behind; N-l-b, non-left-behind.

immunisation rate, suggest a marked inequality between left-behind and non-left-behind children.

Data of children aged 12-72 months $(n=1368)$ were collected in this survey. The immunisation coverage rate of the 1:3:3:3:1 vaccine series in the left-behind and non-left-behind children was $>90 \%$. The goal of immunisation coverage in China for 2005 was set at $90 \%$ for the 1:3:3:3:1 vaccine series. Although the goal was reportedly achieved, we found that the immunisation rates of vaccines, particularly among left-behind children, were lower than the national immunisation rates described by the National Immunization Coverage Survey [13]. Based on the results of surveys in the neighbouring provinces of Hubei, we noted that the five-vaccine allcourse vaccination coverage in left-behind children in the present study $(79.9 \%)$ was lower than that in Hunan $(96.0 \%)$ [19] and higher than that in Hebei [20] and Anhui (72.7\%) [21]. Moreover, the first dose of these five vaccines was most likely to be administered in an age-appropriate manner, and the age-appropriate immunisation rates decreased by dose. These results were consistent with previous studies, which indicated that high coverage estimates mask the shortfalls in timeliness as well as within- and between-country disparities. Hence, great efforts are needed to improve the age-appropriate immunisation rate of these five vaccines, particularly among leftbehind children. By reviewing the coverage reported in our study, and extrapolating the values to the entire
Hubei Province, we estimate that approximately 10 000 children would not be fully vaccinated. It can also be assumed that there are a greater number of children who do not receive age-appropriate vaccination, compared with that reported currently. By analysing the immunisation coverage rate, we found that the factors associated with the immunisation status for the five-vaccine series were related to parenting style, child's gender and age, socio-economic conditions, terrain and primary caregiver's education and attitude towards vaccination. Compared with children residing with both parents, left-behind children tend to have a higher incidence of missing immunisation. Consistent with most previous surveys [21, 22], children from non-migrant families had better vaccination outcomes as compared with children from migrant families. Moreover, non-left-behind children were more likely to receive age-appropriate immunisation than leftbehind children. Furthermore, in the present study, there was a difference in the birth doses of HepB between both groups of children. We also found that many left-behind children were born in the field by their mothers. In China, newborns are mandated to receive the first dose of $\mathrm{HBV}$ vaccine within $24 \mathrm{~h}$ after their birth in the hospital. Based on this, we think one potential reason for this difference is that a written record of immunisation history was missing during migration, and hence, the child would be regarded as unvaccinated and the immunisation procedure would be restarted when they returned to 
Table 3. Factors associated with the five-vaccine all-course vaccination rate in binary logistic regression analysis

\begin{tabular}{|c|c|c|c|c|c|c|}
\hline & \multicolumn{3}{|c|}{ Immunisation rate } & \multicolumn{3}{|c|}{$\begin{array}{l}\text { Age-appropriate immunisation } \\
\text { rate }\end{array}$} \\
\hline & OR & $95 \% \mathrm{CI}$ & $P$ & OR & $95 \% \mathrm{CI}$ & $P$ \\
\hline \multicolumn{7}{|l|}{ Parenting style } \\
\hline Single-parenting & $0 \cdot 55$ & $0 \cdot 37-0 \cdot 81$ & \multirow[t]{3}{*}{$<0.001$} & $0 \cdot 37$ & $0 \cdot 30-0 \cdot 73$ & \multirow[t]{3}{*}{$<0.001$} \\
\hline Grand-parenting & $0 \cdot 41$ & $0 \cdot 15-0 \cdot 77$ & & $0 \cdot 25$ & $0 \cdot 13-0 \cdot 50$ & \\
\hline Both-parenting & Ref & & & Ref & & \\
\hline \multicolumn{7}{|l|}{ Gender } \\
\hline Boy & Ref & & \multirow[t]{2}{*}{$0 \cdot 250$} & Ref & & \multirow[t]{2}{*}{$0 \cdot 510$} \\
\hline Girl & $0 \cdot 81$ & $0 \cdot 61-1 \cdot 35$ & & $0 \cdot 89$ & $0 \cdot 77-1 \cdot 21$ & \\
\hline \multicolumn{7}{|l|}{ Children age (months) } \\
\hline $12-24$ & Ref & & \multirow[t]{3}{*}{$0 \cdot 031$} & Ref & & \multirow[t]{3}{*}{$0 \cdot 015$} \\
\hline $25-48$ & $0 \cdot 71$ & $0 \cdot 53-0 \cdot 89$ & & $0 \cdot 516$ & $0 \cdot 37-0 \cdot 81$ & \\
\hline 49-72 & $0 \cdot 62$ & $0 \cdot 44-0 \cdot 82$ & & $0 \cdot 308$ & $0 \cdot 27-0.74$ & \\
\hline \multicolumn{7}{|l|}{ Only child } \\
\hline Yes & $0 \cdot 53$ & $0 \cdot 44-0 \cdot 62$ & \multirow[t]{2}{*}{$<0 \cdot 001$} & $0 \cdot 46$ & $0 \cdot 32-0 \cdot 62$ & \multirow[t]{2}{*}{$<0 \cdot 001$} \\
\hline $\mathrm{No}$ & Ref & & & Ref & & \\
\hline \multicolumn{7}{|l|}{ Caregiver's education } \\
\hline No schooling & Ref & & \multirow[t]{4}{*}{$<0.001$} & Ref & & \multirow[t]{4}{*}{$<0 \cdot 001$} \\
\hline Elementary school & $1 \cdot 14$ & $0 \cdot 69-1 \cdot 41$ & & $1 \cdot 21$ & $0 \cdot 78-1 \cdot 97$ & \\
\hline Middle school & $2 \cdot 23$ & $2 \cdot 01-3 \cdot 57$ & & $2 \cdot 15$ & $1 \cdot 19-5 \cdot 36$ & \\
\hline High school and above & $3 \cdot 15$ & $1 \cdot 37-5 \cdot 13$ & & $2 \cdot 89$ & $1 \cdot 62-6 \cdot 05$ & \\
\hline \multicolumn{7}{|l|}{ Caregiver's age (years) } \\
\hline$<30$ & Ref & & \multirow[t]{4}{*}{$0 \cdot 751$} & Ref & & \multirow[t]{4}{*}{$0 \cdot 574$} \\
\hline $31-40$ & $0 \cdot 89$ & $0 \cdot 65-1 \cdot 20$ & & $1 \cdot 01$ & $0 \cdot 72-1 \cdot 23$ & \\
\hline $41-50$ & $0 \cdot 91$ & $0 \cdot 78-1 \cdot 35$ & & $1 \cdot 12$ & $0 \cdot 84-1 \cdot 46$ & \\
\hline$>51$ & $0 \cdot 73$ & $0 \cdot 46-1 \cdot 75$ & & $0 \cdot 85$ & $0 \cdot 37-1 \cdot 75$ & \\
\hline \multicolumn{7}{|c|}{ Annual household income (Yuan per year) } \\
\hline$<5000$ & $0 \cdot 49$ & $0 \cdot 31-0 \cdot 67$ & \multirow[t]{3}{*}{$0 \cdot 021$} & $0 \cdot 32$ & $0 \cdot 11-0 \cdot 65$ & \multirow[t]{3}{*}{$<0 \cdot 001$} \\
\hline $5000-8000$ & $0 \cdot 78$ & $0 \cdot 54-0 \cdot 86$ & & $0 \cdot 45$ & $0 \cdot 23-0 \cdot 79$ & \\
\hline$>8000$ & Ref & & & Ref & & \\
\hline \multicolumn{7}{|l|}{ Terrain } \\
\hline Plain area & Ref & & $0 \cdot 037$ & Ref & & $<0 \cdot 001$ \\
\hline Hilly area & $0 \cdot 80$ & $0.65-0.99$ & & $0 \cdot 75$ & $0 \cdot 56-0.95$ & \\
\hline Mountainous area & $0 \cdot 62$ & $0 \cdot 41-0 \cdot 94$ & & $0 \cdot 54$ & $0 \cdot 24-0 \cdot 89$ & \\
\hline Awareness of the importar & & & & & & \\
\hline No & $0 \cdot 76$ & $0 \cdot 53-0.97$ & $0 \cdot 028$ & $0 \cdot 45$ & $0 \cdot 25-0.79$ & $<0 \cdot 001$ \\
\hline Yes & Ref & & & Ref & & \\
\hline Knowledge of the vaccina & & & & & & \\
\hline No & $0 \cdot 69$ & $0 \cdot 49-0.96$ & $0 \cdot 032$ & $0 \cdot 23$ & $0 \cdot 11-0 \cdot 53$ & $<0 \cdot 001$ \\
\hline Yes & Ref & & & Ref & & \\
\hline Satisfied with the immunis & & & & & & \\
\hline No & $0 \cdot 85$ & $0 \cdot 61-1 \cdot 21$ & $0 \cdot 79$ & $0 \cdot 82$ & $0 \cdot 57-1 \cdot 35$ & $0 \cdot 56$ \\
\hline Yes & Ref & & & Ref & & \\
\hline
\end{tabular}

their home villages. Therefore, the age-appropriate immunisation coverage for the first dose of the HBV vaccine series was most likely to be underestimated.

However, in 2013, approximately 65 million children were 'left behind' in rural areas, and most were raised by their grandparents. It is noteworthy that the main cause for incomplete vaccination is the lack of an individual to take the child to the immunisation station. A survey of individuals in the Hubei Province shows that left-behind children have poor living conditions, lag in educational attainment, insecurity and difficultly in communication [23]. Moreover, the left-behind children may have reduced sources of information and a lower acceptance of medical care in general, and preventive health services in particular. 
In the present study, the primary caregiver education level and awareness of the importance of immunisation were significantly associated with the children's immunisation status. Parental knowledge of vaccination may lead to improved sustainable management of a child's immunisation at the appropriate age. As most primary caregivers of left-behind children were grandparents who did not complete middle school, they were unware of the importance of immunisation to the child's health, and hence, they did not precisely follow the immunisation schedules. The current results indicated that a higher level of awareness of vaccination in the primary caregiver led to better vaccination status among children in rural China. This suggests that improving caregiver knowledge and practices may help improve a child's immunisation rate. Interventions for caregivers have been proven to be effective for improving the vaccination outcomes of preschool children in China [18]. Hence, these interventions are urgently required for younger children who are left behind. Based on the answers to questions of awareness of the importance of immunisation and knowledge of the vaccination programme in our survey, we found that, compared with parents, grandparents had a relatively lower level of vaccination-related health literacy, and that a caregiver's age was inversely correlated with health literacy. These findings are consistent with the results of a previous study, which indicated that targeted interventions should specifically meet the needs of non-parent caregivers [24].

Consistent with previous studies $[25,26]$, the geographic region of the present study had a significant effect on the childhood full immunisation status. The proportion of fully vaccinated children was higher in plain areas than in mountainous and hilly areas. First, the children who live in poor rural areas, usually in mountainous areas, are very difficult to reach, particularly for health services. Second, it is known that health resources are unevenly distributed in different areas; high healthcare quality resources are primarily concentrated in urban areas and are relatively scarce in rural areas. Vaccination services are provided daily in cities; however, children in mountainous areas are usually vaccinated at an immunisation station on a single day in the month.

In addition, family income is significantly correlated with the vaccination status in children. The vaccination coverage and timeliness of vaccination of the five-vaccine series among the children from highincome families were better than those among children in low-income families. We hypothesised that the caregivers are under pressure to receive income, and are hence too busy to take children to receive vaccines. Accordingly, we found that women and children from low-income families were at a disadvantage in terms of access to healthcare in rural China [27].

Similar to other studies [28] there was no significant gender-related difference in immunisation in the present study. This phenomenon suggests that there is no sex-related bias in vaccination. However, compared with children who were an only child, those who were not the only child had markedly worse vaccination status. A major reason was that parents in China pay more attention to the care of children in families with one child than in families with many children. This finding is consistent with previous studies [29, 30], wherein children born to parents with two or three children were found to have a lower likelihood of receiving full vaccination as compared with children born to families with only one child.

In China, the government conducts immunisation programmes as social public welfare undertakings. Hence, the government should increase the investment in the programme and offer policy support to cover the immunisation programme and personnel expenses. It is also necessary to strengthen policy coordination among various departments, actively mobilise the participation of the civil society, and expand the coverage of the public health service system. The government should also pay more attention to the management of left-behind children, and enhance the standards and system of immunisation services. Vaccination inspection of enrolled children and improvement of the revaccination service model in unvaccinated school children are essential to implement revaccination services [31]. The developments made in the information age should be embraced, and measures to broaden the public knowledge of preventive immunisation, establish immunisation programmes via the short message service (SMS) platform, and send reminder messages to caregivers whose children drop out from vaccination, should be initiated. In particular, the SMS platform is an appropriate method for reminding parents in mountainous areas to take their children for vaccination. Vaccination clinics are the primary location for immunisation, and the building of standardised vaccination clinics is important to improve vaccination rates as well as the satisfaction of patients with the vaccination clinics [32]. In the present survey, we found that some parents were still concerned about adverse reactions to vaccination. First, vaccines need to be stored and managed 
appropriately, or they would lose potency and become less effective. Second, the knowledge of vaccines should be imparted routinely to ensure that a greater number of individuals become aware that vaccines used as part of China's EPI are safe and effective, and that these vaccines have eliminated polio and neonatal tetanus and reduced vaccine-preventable diseases to very low levels in China. During 2010-2014, the incidence of hepatitis B was 110 per 100000 whereas that of tuberculosis was 78 per $100000[9$, 33]. Considering the increase in the number of children requiring vaccination, the creation of vaccination centre with electronic information system is important to ensure an appropriate quality of immunisation and an increase in the immunisation rate. Moreover, healthcare education should be amended to convey the benefits of the NIP policy and vaccination-related knowledge to the masses. Last but not least, the national immunisation research network should be established to seek to implement an effective surveillance method to measure immunisation coverage and adverse events to provide correct information to public health authorities in time [34].

\section{ACKNOWLEDGEMENTS}

This study did not receive any financial support.

\section{AUTHOR CONTRIBUTIONS}

Z.L.N., X.D.T., H.Y.S. and Y.W. contributed to the conception, design and interpretation of data. Z.L.N. and X.D.T. wrote the manuscript. All authors read and approved the final manuscript.

\section{DECLARATION OF INTEREST}

None.

\section{REFERENCES}

1. Su S, et al. Psychological adjustment among left-behind children in rural China: the role of parental migration and parent-child communication. Child: Care, Health and Development 2013; 39: 162-170.

2. Wen M, et al. Positive youth development in rural China: the role of parental migration. Social Science \& Medicine 2015; 132: 261-269.

3. Biao X, et al. How far are the left-behind left behind? A preliminary study in rural China. Population, Space and Place 2007; 13: 179-191.

4. Wen M, et al. Child development in rural China: children left behind by their migrant parents and children of nonmigrant families. Child Development 2012; 83: $120-136$.

5. Zhao X, et al. Left-behind children in rural China experience higher levels of anxiety and poorer living conditions. Acta Paediatrica 2014; 103: 665-670.

6. Cui FQ, et al. Immunization coverage and its determinants in children aged 12-23 months in Gansu, China. Vaccine 2007; 25: 664-671.

7. Black RE, et al. Global, regional, and national causes of child mortality in 2008: a systematic analysis. Lancet 2010; 375: 1969-1987.

8. Schuller KA, et al. Factors associated with influenza vaccination among US children in 2008. Journal of Infection and Public Health 2013; 6: 80-88.

9. Gellin BG, et al. Do parents understand immunizations? A national telephone survey. Pediatrics 2000; 106: 1097-1102.

10. Babirye JN, et al. Timeliness of childhood vaccinations in Kampala Uganda: a community-based crosssectional study. PLOS ONE 2012; 7: e35432.

11. Wiysonge CS, et al. Individual and contextual factors associated with low childhood immunisation coverage in sub-Saharan Africa: a multilevel analysis. PLoS ONE 2012; 7: e37905.

12. Sun M, et al. Immunization status and risk factors of migrant children in densely populated areas of Beijing, China. Vaccine 2010; 28: 1264-1274.

13. Cao L, et al. National immunization coverage survey in China after integrated more vaccines into EPI since 2008. Chinese Journal of Vaccines and Immunization 2008; 5: 419-424.

14. World Health Organization (WHO). Immunization Coverage Cluster Survey-Reference Manual. Geneva, Switzerland: World Health Organization, 2005.

15. Xu Z, et al. Evaluation of measles catch-up immunization campaign in Hubei, 2010. Chinese Journal of Viral Diseases 2011; 3: 208-211.

16. Yao XP, et al. Epidemiological characteristics of measles in Hubei Province, 2006-2012. Chinese Journal of Child Health Care 2014; 7: 683-686.

17. Zheng L, et al. Epidemiological characteristics of mumps in Hubei Province, 2005-2015. Chinese Journal of Vaccines and Immunization 2006; 3: 263-266.

18. Kusuma YS, et al. Migration and immunization: determinants of childhood immunization uptake among socioeconomically disadvantaged migrants in Delhi, India. Tropical Medicine \& International Health 2010; 11: $1326-1332$.

19. Liu H, et al. Survey on the immunization coverage rates and countermeasures among left-behind children aged 1-6 years old in Changsha rural areas. Chinese Journal of Disease Control \& Prevention 2014; 18: 545-548.

20. Guo YC, et al. Survey of inoculation rates of left-behind children in Wuji County of Shijiazhuang. Modern Preventive Medicine 2014; 23: 4257-4277.

21. Lu ZJ, et al. Analysis on basic vaccination situation of left-behind children in rural areas in Anhui and Sichuan Provinces. Chinese Journal of Vaccines and Immunization 2009; 1: 61-64. 
22. Liu C, et al. Influence of parental migration to hepatitis $B$ vaccination among left-behind children in Hebei province. China Journal of Public Health 2014; 30: 861-863.

23. Wang YJ, et al. Preliminary study on the health status among the "left-behind" children in the Xiantao rural area of Hubei Province. Chinese Journal of Contemporary Pediatrics 2011; 12: 977-980.

24. He G, et al. Depression in left-behind elderly in rural China: prevalence and associated factors. Geriatrics \& Gerontology International 2016; 16: 638-643.

25. Mathew JL, et al. Inequity in childhood immunization in India: a systematic review. Indian Pediatrics 2012; 49: 203-223.

26. Quansah E, et al. Social factors influencing child health in Ghana. PLoS ONE 2006; 11: e0145401.

27. Li JH, et al. Gender inequality, family planning, and maternal and child care in a rural Chinese county. Social Science \& Medicine 2004; 59: 695-708.

28. Abeykoon AT. Sex preference in South Asia: Sri Lanka an outlier. Asia-Pacific Population Journal/United Nations 1995; 10(3): 5-16.
29. Luman ET, et al. Maternal characteristics associated with vaccination of young children. Pediatrics 2003; 111S: $1215-1218$.

30. Abadura SA, et al. Individual and community level determinants of childhood full immunization in Ethiopia: a multilevel analysis. BMC Public Health 2015; 15: 972.

31. Butsashvili M, et al. Associated factors for recommending $\mathrm{HBV}$ vaccination to children among Georgian health care workers. BMC Infection Disease 2012; 12: $1-6$.

32. Zhang $\mathbf{P}$, et al. Analysis of epidemiological characteristics and incidence trend of hepatitis B in Hubei Province (2010-2014). Journal of Public Health and Prevention Medicine 2016; 1: 33-35.

33. Yi SY, et al. Epidemiological characteristics of tuberculosis in Yiling district of Hubei Province, 2010-2014. Journal of Medical Forum 2014; 7: 683-686.

34. Langley JM, et al. Rapid surveillance for health events following a mass meningococcal $\mathrm{B}$ vaccine program in a university setting: a Canadian immunization research network study. Vaccine 2016; 34: 4046-4049. 